\title{
Editorial
}

Published online: November 16, 2006

\section{Is bcl-2 a Key Molecule in the Abnormal Immune Response of Hemodialysis Patients?}

\author{
A. Martin-Malo ${ }^{a} \quad$ R. Ramirez ${ }^{\text {b }} \quad$ P. Aljama ${ }^{a}$ \\ ${ }^{\mathrm{a}}$ Nephrology Department, ${ }^{\mathrm{b}}$ Research Unit, Reina Sofia University Hospital, Cordoba, Spain
}

Apoptosis plays an important role in the homeostasis of the immune response [1]. In fact, a disturbance in the programmed cell death of immunocompetent cells has been reported in HIV infection, some autoimmune diseases and chronic kidney disease (CKD) that may induce a chronic inflammatory status in these patients [1].

The family of Bcl-2 proteins is involved in the apoptotic machinery. Apoptosis is promoted by several stimuli which are subject to complex regulating processes [2]. In peripheral blood mononuclear cells (PBMC), the process of programmed cell death is primarily regulated by the Fas/Apo-1 and p53 genes, which promote apoptosis, and by bcl-2, a survival-related gene that is the main antiapoptotic molecule of this family of protooncogenes [2]. The identification of key apoptotic mediators remains elusive $[1,2]$.

In uremia, the immunodeficiency response is mainly related to an abnormal cell activation and apoptosis of PBMC [3]. In these cells, the anti-apoptotic molecule bcl-2 may play a crucial role in the regulation of apoptosis. In a recent paper we have reported that there was a decrease in the proportion of Th1 lymphocytes relative to Th2 in CKD patients [4]. It was also shown that Th1 lymphocytes from CKD patients were highly susceptible to undergo apoptosis, which was associated with a low expression of the anti-apoptotic bcl-2 gene. On the other

\section{KARGER}

Fax +4161306 1234

E-Mail karger@karger.ch

www.karger.com (c) 2006 S. Karger AG, Basel

$0253-5068 / 06 / 0246-0540 \$ 23.50 / 0$

Accessible online at:

www.karger.com/bpu hand, the report in this issue of Blood Purification by Koliousi et al. [5] evaluated the effect of a hemodialysis (HD) session using two different types of membrane (cellulosic and synthetic) on in vivo bcl-2 expression of lymphocytes and monocytes. bcl-2 expression increased significantly in lymphocytes after the HD procedure, irrespective of the membrane used. In contrast, the expression of monocyte bcl-2 remained stable throughout the dialysis session. The authors speculated that the decrease in the rate of apoptosis in lymphocytes after the HD session might be related to the correction of uremia and metabolic acidosis, both of which are well-known apoptosis-inducing factors that increase the signals produced by the anti-apoptotic bcl-2 genes. The apoptosis of Th1 lymphocytes induced by uremia may participate in the imbalance of the immune response that is characteristic of CKD patients, and this increase in T-lymphocyte apoptosis is mainly related to a decrease in the expression of bcl-2. In a preliminary study (not yet published) we have observed that a single HD session was able to normalize the Th1/Th2 ratio. The increase in bcl2 expression and the reduction in lymphocyte apoptosis observed at the end of HD session may thus be capable of explaining the normalization of Th1/Th2 ratio in $\mathrm{HD}$ patients.

There have been several recent reports that many drugs that are usually used in the treatment of CKD pa- 
tients, such as statins, angiotensin-converting enzyme inhibitors, angiotensin receptor antagonists and erythropoietin may prevent apoptosis, regulating the anti-apoptotic effect of bcl-2. Further studies are needed in order to determine whether the use of these therapeutic agents and/or the increase of the dialysis frequency are capable of improving or normalizing the abnormal immune response observed in CKD patients.

A better understanding of how PBMC apoptosis is regulated by bcl-2 in HD patients may help us to develop new dialysis strategies aimed at reducing the high mortality rate in the dialysis population.

\section{References}

1 Kuwana T, Newmeyer DD: bcl-2 family proteins and the role of mitochondria in apoptosis. Curr Opin Cell Biol 2003;15:691-699.

2 Antonsson B: Mitochondria and Bcl-2 family proteins in apoptosis signalling pathways. Mol Cell Biochem 2004;257:141-155.

3 Martin-Malo A, Carracedo J, Ramirez R, Rodriguez-Benot R, Soriano S, Rodriguez M, Aljama P: Effect of uremia and dialysis modality on mononuclear cell apoptosis. J Am Soc Nephrol 2000;11:936-942.
4 Alvarez-Lara MA, Carracedo J, Ramirez R, Martin-Malo A, Rodriguez M, Madueño JA, Aljama P: The imbalance in the ratio of Th1 and Th2 helper lymphocytes in uremia is mediated by an increased apoptosis of Th1 subset. Nephrol Dial Transplant 2004;19: 3084-3090.

5 Koliousi E, Vartholomatos G, Katopodis KP, Kolaitis N, Siamopoulos KC: Effect of the hemodialysis session on bcl-2 expression in peripheral blood mononuclear cells in vivo. Blood Purif 2006;24:542-547. 